\title{
ON THE HYERS-ULAM-RASSIAS STABILITY OF THE JENSEN EQUATION IN DISTRIBUTIONS
}

\author{
Eun Gu Lee and Jaeyoung Chung
}

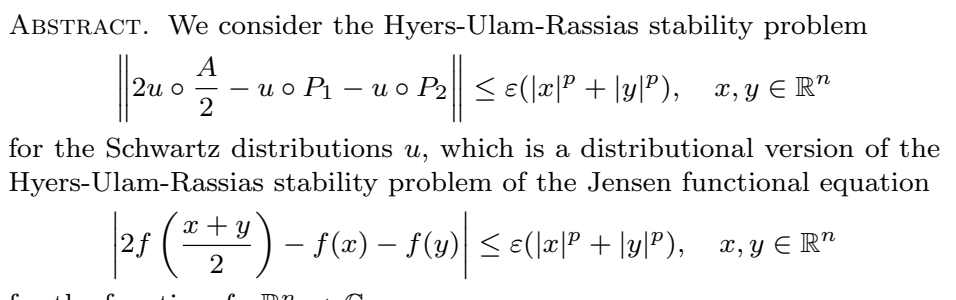
for the function $f: \mathbb{R}^{n} \rightarrow \mathbb{C}$.

\section{Introduction}

The stability problems of functional equations go back to 1940 when S. M. Ulam proposed the following problem [24]:

Let $f$ be a mapping from a group $G_{1}$ to a metric group $G_{2}$ with metric $d(\cdot, \cdot)$ such that

$$
d(f(x y), f(x) f(y)) \leq \varepsilon .
$$

Then does there exist a group homomorphism $h$ and $\delta_{\epsilon}>0$ such that

$$
d(f(x), h(x)) \leq \delta_{\epsilon}
$$

for all $x \in G_{1}$

This problem was solved affirmatively by D. H. Hyers under the assumption that $G_{2}$ is a Banach space (see Hyers [12]). Since then, the stability problems of many other functional equations have been investigated $[1,2,3,4,5,6,7$, $9,10,16,17,18,19,20,21,22]$. Among them, generalizing the well known stability theorem of D. H. Hyers, Th. M. Rassias [22] and Z. Gajda [9] showed the following stability theorem for the Cauchy equation:

Received April 2, 2010; Revised August 25, 2010.

2010 Mathematics Subject Classification. 39B82, 46F99.

Key words and phrases. stability, Gauss transforms, heat kernel, distributions, tempered distribution, Jensen functional equation. 
Theorem 1.1 ([9,22]). Let $f$ be a mapping from a normed linear space $X$ to a Banach space $Y$ satisfying the inequality

$$
\|f(x+y)-f(x)-f(y)\| \leq \epsilon\left(\|x\|^{p}+\|y\|^{p}\right), \quad p \neq 1
$$

for all $x, y \in X(x \neq 0$ and $y \neq 0$ if $p<0)$. Then there exists a unique function $g: X \rightarrow Y$ satisfying

$$
g(x+y)-g(x)-g(y)=0
$$

such that

$$
\|f(x)-g(x)\| \leq \frac{2 \varepsilon}{\left|2^{p}-2\right|}\|x\|^{p}
$$

for all $x \in X(x \neq 0$ if $p<0)$.

As a similar result, generalizing the Hyers-Ulam stability theorem for the Jensen functional equation of K. Kominek [14], S. M Jung [13] prove a HyersUlam-Rassias stability theorem for the Jensen functional equation

$$
\left|2 f\left(\frac{x+y}{2}\right)-f(x)-f(y)\right| \leq \epsilon\left(\|x\|^{p}+\|y\|^{p}\right) .
$$

For more interesting results related to the Hyers-Ulam stability of Jensen functional equation we refer the reader to the results of J.-H. Bae, D.-O. Lee and W.-G. Park [1] and that of C.-G. Park [16, 17, 18] and C.-G. Park and W.-G. Park $[19,20]$.

In this paper, we consider the stability theorem for the Jensen functional equation (1.2) in the spaces of generalized functions such as the spaces $\mathcal{S}^{\prime}$ and $\mathcal{D}^{\prime}$ of tempered distributions and distributions of L. Schwartz, respectively. Making use of the pullbacks of generalized function we extend the inequality (1.2) to distributions $u$ as follows:

$$
\left\|2 u \circ \frac{A}{2}-u \circ P_{1}-u \circ P_{2}\right\| \leq \varepsilon\left(|x|^{p}+|y|^{p}\right)
$$

for even integers $p \geq 2$, where $A(x, y)=x+y, P_{1}(x, y)=x, P_{2}(x, y)=$ $y, x, y \in \mathbb{R}^{n}$, and $u \circ A, v \circ P_{1}$ and $w \circ P_{2}$ are the pullbacks of $u, v, w$ by $A, P_{1}$ and $P_{2}$, respectively. Also $|\cdot|$ denotes the Euclidean norm and the inequality $\|\cdot\| \leq \psi(x, y)$ in (1.3) means that $|\langle\cdot, \varphi\rangle| \leq\|\psi \varphi\|_{L^{1}}$ for all test functions $\varphi \in C_{c}^{\infty}\left(\mathbb{R}^{2 n}\right)$ which will be introduced in Section 2 .

As the main result, we prove the following: Let $u \in \mathcal{D}^{\prime}$ satisfy

$$
\left\|2 u \circ \frac{A}{2}-u \circ P_{1}-u \circ P_{2}\right\| \leq \epsilon\left(|x|^{2 p}+|y|^{2 p}\right)
$$

for some integer $p>1$. Then there exist a unique $a \in \mathbb{C}^{n}$ and $c \in \mathbb{C}$ such that

$$
\|u-a \cdot x-c\| \leq \frac{2 \epsilon}{4^{p}-2}|x|^{2 p} .
$$




\section{Schwartz distributions}

We briefly introduce the space $\mathcal{D}^{\prime}\left(\mathbb{R}^{n}\right)$ of distributions and the space $\mathcal{S}^{\prime}\left(\mathbb{R}^{n}\right)$ of tempered distributions. Here we use the multi-index notations, $|\alpha|=\alpha_{1}+$ $\cdots+\alpha_{n}, \alpha !=\alpha_{1} ! \cdots \alpha_{n} !, x^{\alpha}=x_{1}^{\alpha_{1}} \cdots x_{n}^{\alpha_{n}}$ and $\partial^{\alpha}=\partial_{1}^{\alpha_{1}} \cdots \partial_{n}^{\alpha_{n}}$ for $x=$ $\left(x_{1}, \ldots, x_{n}\right) \in \mathbb{R}^{n}, \alpha=\left(\alpha_{1}, \ldots, \alpha_{n}\right) \in \mathbb{N}_{0}^{n}$, where $\mathbb{N}_{0}$ is the set of non-negative integers and $\partial_{j}=\frac{\partial}{\partial x_{j}}$. We also denote by $C_{c}^{\infty}\left(\mathbb{R}^{n}\right)$ the set of all infinitely differentiable functions on $\mathbb{R}^{n}$ with compact supports.

Definition 2.1. A distribution $u$ is a linear form on $C_{c}^{\infty}\left(\mathbb{R}^{n}\right)$ such that for every compact set $K \subset \mathbb{R}^{n}$ there exist constants $C>0$ and $k \in \mathbb{N}_{0}$ such that

$$
|\langle u, \varphi\rangle| \leq C \sum_{|\alpha| \leq k} \sup \left|\partial^{\alpha} \varphi\right|
$$

for all $\varphi \in C_{c}^{\infty}\left(\mathbb{R}^{n}\right)$ with supports contained in $K$. The set of all distributions is denoted by $\mathcal{D}^{\prime}\left(\mathbb{R}^{n}\right)$.

Definition 2.2. We denote by $\mathcal{S}$ or $\mathcal{S}\left(\mathbb{R}^{n}\right)$ the Schwartz space of all infinitely differentiable functions $\varphi$ in $\mathbb{R}^{n}$ such that

$$
\|\varphi\|_{\alpha, \beta}=\sup _{x}\left|x^{\alpha} \partial^{\beta} \varphi(x)\right|<\infty
$$

for all $\alpha, \beta \in \mathbb{N}_{0}^{n}$, equipped with the topology defined by the seminorms $\|\cdot\|_{\alpha, \beta}$. The elements of $\mathcal{S}$ are called rapidly decreasing functions and the elements of the dual space $\mathcal{S}^{\prime}$ are called tempered distributions.

It is well known that the following topological inclusions:

$$
C_{c}^{\infty} \hookrightarrow \mathcal{S}, \quad \mathcal{S}^{\prime} \hookrightarrow \mathcal{D}^{\prime}
$$

Example 2.1 ([11, 23]). In the usual sense of differentiations, the derivatives of locally integrable functions make no sense, however, one can differentiate every locally integrable function in the space of Schwartz distributions. As a matter of fact, it is well known that every derivative $\partial^{\alpha} f$ of a locally integrable function $f: \mathbb{R}^{n} \rightarrow \mathbb{C}$ defines a distribution via the relation

$$
\left\langle\partial^{\alpha} f, \varphi\right\rangle=(-1)^{|\alpha|} \int_{\mathbb{R}^{n}} f(x) \partial^{\alpha} \varphi(x) d x, \quad \varphi \in C_{c}^{\infty}\left(\mathbb{R}^{n}\right) .
$$

Also it is well known that every derivative $\partial^{\alpha} f$ of locally integrable function $f: \mathbb{R}^{n} \rightarrow \mathbb{C}$ defines a distribution via the relation (2.2) provided that $f$ satisfies the growth condition; there exist positive constants $C$ and $N$ such that

$$
|f(x)| \leq C(1+|x|)^{N}
$$

for all $x \in \mathbb{R}^{n}$.

Example $2.2([11,23])$. Let $H$ be the Heaviside function on $\mathbb{R}$ defined by $H(x)=1$ for $x \geq 0$ and $H(x)=0$ for $x<0$. Then it is easy to see that $H^{\prime}=\delta$ where $\delta$ denotes the Dirac measure on $\mathbb{R}$ which is defined by

$$
\langle\delta, \varphi\rangle=\varphi(0), \quad \varphi \in C_{c}^{\infty}(\mathbb{R}) .
$$


Also every finite sum $u=\sum_{|\alpha| \leq m} \partial^{\alpha} \delta$ of derivatives of $\delta$ defines a tempered distribution.

We denote by $\Omega_{j}$ open subsets of $\mathbb{R}^{n_{j}}$ for $j=1,2$, with $n_{1} \geq n_{2}$.

Definition 2.3. Let $u_{j} \in \mathcal{D}^{\prime}\left(\Omega_{j}\right)$ and $\lambda: \Omega_{1} \rightarrow \Omega_{2}$ be a smooth function such that for each $x \in \Omega_{1}$ the derivative $\lambda^{\prime}(x)$ is surjective, that is, the Jacobian matrix $\nabla \lambda$ of $\lambda$ has rank $n_{2}$. Then there exists a unique continuous linear map $\lambda^{*}: \mathcal{D}^{\prime}\left(\Omega_{2}\right) \rightarrow \mathcal{D}^{\prime}\left(\Omega_{1}\right)$ such that $\Lambda^{*} u=u \circ \lambda$ when $u$ is a continuous function. We call $\lambda^{*} u$ the pullback of $u$ by $\lambda$ and often denoted by $u \circ \lambda$.

In particular if $\lambda$ is a diffeomorphism (a bijection with $\lambda, \lambda^{-1}$ smooth functions) the pullback $u \circ \lambda$ can be written as follows:

$$
\langle u \circ \lambda, \varphi\rangle=\left\langle u,\left(\varphi \circ \lambda^{-1}\right)(x)\right|\left(\nabla \lambda^{-1}(x)|\rangle .\right.
$$

As a matter of fact, the pullbacks $u \circ A, u \circ P_{1}, u \circ P_{2}$ can be written in a transparent way as

$$
\begin{gathered}
\langle u \circ A, \varphi(x, y)\rangle=\left\langle u, \int \varphi(x-y, y) d y\right\rangle, \\
\left\langle u \circ P_{1}, \varphi(x, y)\right\rangle=\left\langle u, \int \varphi(x, y) d y\right\rangle, \\
\left\langle u \circ P_{2}, \varphi(x, y)\right\rangle=\left\langle u, \int \varphi(x, y) d x\right\rangle
\end{gathered}
$$

for all test functions $\varphi \in \mathcal{S}\left(\mathbb{R}^{2 n}\right)$.

We refer the reader to ([11], chapter VI) for pullbacks of distributions and to $[11,23]$ for more details of distributions and tempered distributions.

\section{Main theorems}

We denote by $\delta(x)$ the function on $\mathbb{R}^{n}$,

$$
\delta(x)= \begin{cases}A \exp \left(-\frac{1}{\sqrt{1-|x|^{2}}}\right), & |x|<1 \\ 0, & |x| \geq 1\end{cases}
$$

where

$$
A=\left(\int_{|x|<1} \exp \left(-\frac{1}{\sqrt{1-|x|^{2}}}\right) d x\right)^{-1} .
$$

It is easy to see that $\delta(x)$ is an infinitely differentiable function with support $\{x:|x| \leq 1\}$. We employ the regularizing function $\delta_{t}(x):=t^{-n} \delta(x / t), t>0$. Let $u \in \mathcal{D}^{\prime}$. Then, for each $t>0,\left(u * \delta_{t}\right)(x)=\left\langle u_{y}, \delta_{t}(x-y)\right\rangle$ is a smooth function of $x \in \mathbb{R}^{n}$ and $\left(u * \delta_{t}\right)(x) \rightarrow u$ as $t \rightarrow 0^{+}$in the sense that

$$
\lim _{t \rightarrow 0^{+}} \int\left(u * \delta_{t}\right)(x) \varphi(x) d x=\langle u, \varphi\rangle
$$

for all $\varphi \in C_{c}^{\infty}$. 
Lemma 3.1. Let $u \in \mathcal{D}^{\prime}$ satisfy the inequality

$$
\left\|2 u \circ \frac{A}{2}-u \circ P_{1}-u \circ P_{2}\right\| \leq \epsilon\left(|x|^{2 p}+|y|^{2 p}\right)
$$

for some integer $p>1$. Then $u \in \mathcal{S}^{\prime}$.

Proof. We denote by

$$
\Psi(x, y, t, s)=\epsilon\left(|\xi|^{2 p} * \delta_{t}(\xi)\right)(x)+\epsilon\left(|\eta|^{2 p} * \delta_{s}(\eta)\right)(y) .
$$

Convolving $\delta_{t}(x) \delta_{s}(y)$ in each side of (3.1) the inequality (3.1) is converted to the following stability problem

$$
\left|\left(u^{*} * \delta_{t} * \delta_{s}\right)(x+y)-\left(u * \delta_{t}\right)(x)-\left(u * \delta_{s}\right)(y)\right| \leq \Psi(x, y, t, s)
$$

for $x, y \in \mathbb{R}^{n}, t, s>0$, where $\left\langle u^{*}, \varphi(x)\right\rangle=2^{n+1}\langle u, \varphi(2 x)\rangle$. From (3.2) it is easy to see that

$$
f(x):=\limsup _{t \rightarrow 0^{+}}\left(u * \delta_{t}\right)(x)
$$

exists. Letting $y=0$ in $(3.2)$ we have

$$
\left|\left(u^{*} * \delta_{t} * \delta_{s}\right)(x)-\left(u * \delta_{t}\right)(x)-\left(u * \delta_{s}\right)(0)\right| \leq \Psi(x, 0, t, s)
$$

for $x \in \mathbb{R}^{n}, t, s>0$. From (3.2) and (3.3) we have

$$
\begin{aligned}
& \left|\left(u * \delta_{t}\right)(x+y)-\left(u * \delta_{t}\right)(x)-\left(u * \delta_{s}\right)(y)+\left(u * \delta_{s}\right)(0)\right| \\
\leq & \Psi(x, y, t, s)+\Psi(x+y, 0, t, s)
\end{aligned}
$$

for $x, y \in \mathbb{R}^{n}, t, s>0$. Letting $s \rightarrow 0^{+}$so that $\left(u * \delta_{s}\right)(y) \rightarrow f(y)$ in (3.4) we have

$$
\begin{aligned}
& \left|\left(u * \delta_{t}\right)(x+y)-\left(u * \delta_{t}\right)(x)-f(y)+f(0)\right| \\
\leq & \Psi\left(x, y, t, 0^{+}\right)+\Psi\left(x+y, 0, t, 0^{+}\right)
\end{aligned}
$$

for $x, y \in \mathbb{R}^{n}, t, s>0$. Putting $x=0$ and letting $t \rightarrow 0^{+}$so that $\left(u * \delta_{t}\right)(0) \rightarrow$ $f(0)$ in (3.5) we have

$$
\|u-f(y)\| \leq 2 \epsilon|y|^{2 p} .
$$

On the other hand, let

$$
D(x, y, t)=\left(u * \delta_{t}\right)(x+y)-\left(u * \delta_{t}\right)(x)-f(y)+f(0) .
$$

Then we have

$$
\begin{aligned}
|f(x+y)-f(x)-f(y)+f(0)| \leq & |D(x, y, t)|+|-D(0, x+y, t)|+|D(0, x, t)| \\
\leq & \Psi\left(x, y, t, 0^{+}\right)+\Psi\left(x+y, 0, t, 0^{+}\right) \\
& +\Psi\left(0, x+y, t, 0^{+}\right)+\Psi\left(x+y, 0, t, 0^{+}\right) \\
& +\Psi\left(0, x, t, 0^{+}\right)+\Psi\left(x, 0, t, 0^{+}\right)
\end{aligned}
$$

for all $x, y \in \mathbb{R}^{n}, t>0$. Letting $t \rightarrow 0^{+}$in the above inequality we have

$$
|f(x+y)-f(x)-f(y)+f(0)| \leq 3 \epsilon|x+y|^{2 p}+3 \epsilon|x|^{2 p}+\epsilon|y|^{2 p} .
$$


By the results in $[9,10]$, there exists a unique function $A$ satisfying

$$
A(x+y)=A(x)+A(y)
$$

such that

$$
|f(x)-A(x)-f(0)| \leq \frac{\epsilon\left(3 \cdot 4^{p}+4\right)}{4^{p}-2}|x|^{2 p} .
$$

Indeed, let $F(x)=f(x)-f(0)$. Then $A$ is given by a locally uniform limit of the sequence of the continuous functions $A_{m}(x)=2^{n} F\left(2^{-n} x\right)$. Thus $A$ is a continuous function. Thus the solution $A$ of the Cauchy functional equation (3.8) has the form $A(x)=a \cdot x$ for some $a \in \mathbb{C}^{n}$. Now, from (3.6) and (3.9) we have

$$
\|u-a \cdot x-f(0)\| \leq K|x|^{2 p},
$$

where $K=\frac{5 \cdot 4^{p} \epsilon}{4^{p}-2}$. It follows from (3.10) that $u$ is a locally integrable function satisfying

$$
|u(x)| \leq|a \cdot x|+|f(0)|+K|x|^{2 p} .
$$

Thus $u \in \mathcal{S}^{\prime}$. This completes the proof.

Now we may employ the $n$-dimensional heat kernel $E_{t}(x)$ given by

$$
E_{t}(x)=(4 \pi t)^{-n / 2} \exp \left(-|x|^{2} / 4 t\right), x \in \mathbb{R}^{n}, t>0 .
$$

It is easy to see that the heat kernel $E_{t}(\cdot)$ belongs to the Schwartz space $\mathcal{S}\left(\mathbb{R}^{n}\right)$ for each $t>0$. Let $u \in \mathcal{S}^{\prime}$. Then its Gauss transform

$$
\tilde{u}(x, t)=\left(u * E_{t}\right)(x)=\left\langle u_{y}, E_{t}(x-y)\right\rangle, \quad x \in \mathbb{R}^{n}, t>0,
$$

is well defined. As a matter of fact the following result holds [10]:

Lemma $3.2([15])$. Let $u \in \mathcal{S}^{\prime}\left(\mathbb{R}^{n}\right)$. Then its Gauss transform $\tilde{u}(x, t)$ is a $C^{\infty}$-solution of the heat equation satisfying:

(i) There exist positive constants $C, M, N$ and $\delta$ such that

$$
|\tilde{u}(x, t)| \leq C t^{-M}(1+|x|)^{N} \quad \text { in } \quad \mathbb{R}^{n} \times(0, \delta),
$$

(ii) $\tilde{u}(x, t) \rightarrow u$ as $t \rightarrow 0^{+}$in the sense that for every $\varphi \in \mathcal{S}$,

$$
\langle u, \varphi\rangle=\lim _{t \rightarrow 0^{+}} \int \tilde{u}(x, t) \varphi(x) d x .
$$

Conversely, every $C^{\infty}$-solution $U(x, t)$ of the heat equation satisfying the estimate (3.12) can be uniquely expressed as $U(x, t)=\tilde{u}(x, t)$ for some $u \in \mathcal{S}^{\prime}$.

It is well known that the weak semigroup property of the heat kernel

$$
\left(E_{t} * E_{s}\right)(x)=E_{t+s}(x)
$$

holds for convolution. This semigroup property will be very useful later.

Throughout the paper, we denote by

$$
\mathcal{H}_{2 p}(x, t)=\left[|\xi|^{2 p} * E_{t}(\xi)\right](x, t) .
$$


Since $|x|^{2 p}=\sum_{|\gamma|=p} \frac{p !}{\gamma !} x^{2 \gamma}$ we have

$$
\mathcal{H}_{2 p}(x, t)=\sum_{|\gamma|=p} \frac{p !}{\gamma !}(2 \gamma) ! \sum_{0 \leq \alpha \leq \gamma} \frac{t^{|\alpha|} x^{2 \gamma-2 \alpha}}{\alpha !(2 \gamma-2 \alpha) !} .
$$

Note that if $p=1$ we have

$$
\mathcal{H}_{2 \gamma}(x, t)=|x|^{2}+2 n t
$$

and for $p=1,2, \ldots$

$$
\mathcal{H}_{2 \gamma}(x, 0)=|x|^{2 p} \text {. }
$$

We need the following:

Lemma 3.3. Let $g: \mathbb{R}^{n} \times(0, \infty) \rightarrow \mathbb{C}$ be a continuous function satisfying the inequality

$$
|g(x+y, t+s)-g(x, t)-g(y, s)| \leq \epsilon\left(\mathcal{H}_{2 p}(x, t)+\mathcal{H}_{2 p}(y, s)\right)
$$

for some integer $p>1$. Then there exist unique constants $a \in \mathbb{C}^{n}, b \in \mathbb{C}$ such that

$$
|g(x, t)-a \cdot x-b t| \leq \epsilon \psi_{p}(x, t)
$$

for all $x \in \mathbb{R}^{n}, t>0$, where

$$
\psi_{p}(x, t)=\sum_{|\gamma|=p} \frac{p !}{\gamma !}(2 \gamma) ! \sum_{0 \leq \alpha \leq \gamma} \frac{2^{|\alpha|+1} t^{|\alpha|} x^{2 \gamma-2 \alpha}}{\left(2^{|2 \gamma|}-2^{|\alpha|+1}\right) \alpha !(2 \gamma-2 \alpha) !} .
$$

Proof. We can follow the same approach as in $[22,9]$. Indeed, replacing both $x$ and $y$ by $\frac{x}{2}$, both $t$ and $s$ by $\frac{t}{2}$ in (3.14) we have

$$
\left|g(x, t)-2 g\left(2^{-1} x, 2^{-1} t\right)\right| \leq 2 \epsilon \mathcal{H}_{2 p}\left(2^{-1} x, 2^{-1} t\right)
$$

for all $x \in \mathbb{R}^{n}, t>0$. Making use of the induction argument and triangle inequality we have

$$
\begin{aligned}
\left|g(x, t)-2^{m} g\left(2^{-m} x, 2^{-m} t\right)\right| & \leq \epsilon \sum_{j=1}^{m} 2^{j} \mathcal{H}_{2 p}\left(2^{-j} x, 2^{-j} t\right) \\
& \leq \epsilon \sum_{|\gamma|=p} \frac{p !}{\gamma !}(2 \gamma) ! \sum_{0 \leq \alpha \leq \gamma} a_{m, \alpha} \frac{t^{|\alpha|} x^{2 \gamma-2 \alpha}}{\alpha !(2 \gamma-2 \alpha) !}
\end{aligned}
$$

for all $n \in \mathbb{N}, x \in \mathbb{R}^{n}, t>0$, where $a_{m, \alpha}=2^{|\alpha|+1}\left(1-2^{(|\alpha|-|2 \gamma|+1) m}\right) /\left(2^{|2 \gamma|}-\right.$ $\left.2^{|\alpha|+1}\right)$.

Replacing $x, t$ by $2^{-m} x, 2^{-m} t$, respectively in (3.14) and multiplying $2^{m}$ in the result it follows from $p>1$ that

$$
A_{m}(x, t):=2^{m} g\left(2^{-m} x, 2^{-m} t\right)
$$


is a Cauchy sequence which converges locally uniformly, say to $A(x, t)$. Letting $m \rightarrow \infty$ in $(3.15)$ we have

$$
|g(x, t)-A(x, t)| \leq \epsilon \sum_{|\gamma|=p} \frac{p !}{\gamma !}(2 \gamma) ! \sum_{0 \leq \alpha \leq \gamma} a_{\alpha} \frac{t^{|\alpha|} x^{2 \gamma-2 \alpha}}{\alpha !(2 \gamma-2 \alpha) !}
$$

for all $x \in \mathbb{R}^{n}, t>0$, where $a_{\alpha}=2^{|\alpha|+1} /\left(2^{|2 \gamma|}-2^{|\alpha|+1}\right)$.

Replacing $x, y, t, s$ by $2^{-m} x, 2^{-m} y, 2^{-m} t, 2^{-m} s$ in (3.14), respectively, multiplying $2^{m}$ and letting $m \rightarrow \infty$ it follows from the fact $p>1$ that

$$
A(x+y, t+s)-A(x, t)-A(y, s)=0
$$

for all $x, y \in \mathbb{R}^{n}, t, s>0$. To prove the uniqueness of $A(x, t)$, let $B(x, t)$ be another function satisfying (3.12) and (3.13). Then it follows from (3.16), (3.17) and the triangle inequality that for all $n \in \mathbb{N}$,

$$
\begin{aligned}
|A(x, t)-B(x, t)| & \leq m\left|A\left(\frac{x}{m}, \frac{t}{m}\right)-B\left(\frac{x}{m}, \frac{t}{m}\right)\right| \\
& \leq 2 \epsilon \sum_{|\gamma|=p} \frac{p !}{\gamma !}(2 \gamma) ! m^{1-|\gamma|} \sum_{0 \leq \alpha \leq \gamma} a_{\alpha} \frac{t^{|\alpha|} x^{2 \gamma-2 \alpha}}{\alpha !(2 \gamma-2 \alpha) !}
\end{aligned}
$$

for all $x \in \mathbb{R}^{n}, t>0$. Letting $m \rightarrow \infty$, we have $A(x, t)=B(x, t)$ for all $x \in \mathbb{R}^{n}, t>0$. This proves the uniqueness.

Now it is well known that every continuous solution $A(x, t)$ of the Cauchy equation (1.4) has the form

$$
A(x, t)=a \cdot x+b t
$$

for some $a \in \mathbb{C}^{n}, b \in \mathbb{C}$. Thus we have

$$
|g(x, t)-a \cdot x-b t| \leq \epsilon \sum_{|\gamma|=p} \frac{p !}{\gamma !}(2 \gamma) ! \sum_{0 \leq \alpha \leq \gamma} a_{\alpha} \frac{t^{|\alpha|} x^{2 \gamma-2 \alpha}}{\alpha !(2 \gamma-2 \alpha) !}
$$

for all $x, y \in \mathbb{R}^{n}, t>0$, where $a_{\alpha}=2^{|\alpha|+1} /\left(2^{|2 \gamma|}-2^{|\alpha|+1}\right)$. This completes the proof.

Lemma 3.4. Let $f: \mathbb{R}^{n} \times(0, \infty) \rightarrow \mathbb{C}$ be a continuous function satisfying the inequality

$$
\left|2 f\left(\frac{x+y}{2}, \frac{t+s}{4}\right)-f(x, t)-f(y, s)\right| \leq \epsilon\left(\mathcal{H}_{2 p}(x, t)+\mathcal{H}_{2 p}(y, s)\right)
$$

for some integer $p>1$. Then there exist a unique $a \in \mathbb{C}^{n}$, a unique $b \in \mathbb{C}$ and complex constant $c$ such that

$$
|f(x, t)-a \cdot x-b t-c| \leq 2 \epsilon 4^{p} \psi_{p}(x, t)
$$

for all $x \in \mathbb{R}^{n}, t>0$. 
Proof. Let $F(x, t)=2 f(x / 2, t / 4)$. Then we have

$$
|F(x+y, t+s)-f(x, t)-f(y, s)| \leq \epsilon\left(\mathcal{H}_{2 p}(x, t)+\mathcal{H}_{2 p}(y, s)\right) .
$$

Putting $y=0$ in (3.21), it is easy to see that $c:=\limsup _{s \rightarrow 0^{+}} f(0, s)$ exists. Putting $y=0$ and letting $s \rightarrow 0^{+}$in $(3.21)$ so that $f(0, s) \rightarrow c$ we have

$$
|F(x, t)-f(x, t)-c| \leq \epsilon \mathcal{H}_{2 p}(x, t) .
$$

Now it follows from (3.21) and (3.22) that

$$
|G(x+y, t+s)-G(x, t)-G(y, s)| \leq 2 \epsilon\left(\mathcal{H}_{2 p}(x, t)+\mathcal{H}_{2 p}(y, s)\right),
$$

where $G(x, t)=F(x, t)-2 c$. Thus it follows from Lemma 3.3 that there exist a unique $a \in \mathbb{C}^{n}$, a unique $b \in \mathbb{C}$ such that

$$
|F(x, t)-a \cdot x-b t-2 c| \leq 2 \epsilon \psi_{p}(x, t) .
$$

Replacing $x$ by $2 x, t$ by $4 t$ in (3.24) and dividing by 2 in the result we have $(3.25)$

$$
\begin{aligned}
|f(x, t)-a \cdot x-2 b t-c| & \leq 2 \epsilon\left|\psi_{p}(2 x, 4 t)\right| \\
& \leq 2 \epsilon 4^{p} \sum_{|\gamma|=p} \frac{p !}{\gamma !}(2 \gamma) ! \sum_{0 \leq \alpha \leq \gamma} \frac{2^{|\alpha|+1}}{2^{|2 \gamma|}-2^{|\alpha|+1}} \frac{t^{|\alpha|} x^{2 \gamma-2 \alpha}}{\alpha !(2 \gamma-2 \alpha) !}
\end{aligned}
$$

for all $x \in \mathbb{R}^{n}, t>0$. This completes the proof.

Theorem 3.5. Let $u \in \mathcal{D}^{\prime}$ satisfy

$$
\left\|2 u \circ \frac{A}{2}-u \circ P_{1}-u \circ P_{2}\right\| \leq \epsilon\left(|x|^{2 p}+|y|^{2 p}\right)
$$

for some integer $p>1$. Then there exist a unique $a \in \mathbb{C}^{n}$ and $c \in \mathbb{C}$ such that can be written uniquely in the form

$$
\|u-a \cdot x-c\| \leq 2 \epsilon \frac{4^{p}}{4^{p}-2}|x|^{2 p} .
$$

Proof. Convolving in each side of (3.26) the tensor product $E_{t}(x) E_{s}(y)$ of $n$ dimensional heat kernels we have in view of (2.3), (2.4), (2.5) and the semigroup property (3.13),

$$
\begin{aligned}
{\left[\left(2 u \circ \frac{A}{2}\right) *\left(E_{t}(\xi) E_{s}(\eta)\right)\right](x, y) } & =\left\langle 2^{n+1} u_{\xi}, \int E_{t}(x-2 \xi+\eta) E_{s}(y-\eta) d \eta\right\rangle \\
& =\left\langle 2^{n+1} u_{\xi},\left(E_{t} * E_{s}\right)(x+y-2 \xi)\right\rangle \\
& =\left\langle 2^{n+1} u_{\xi}, E_{t+s}(x+y-2 \xi)\right\rangle \\
& =\left\langle 2^{n+1} u_{\xi}, 2^{-n} E_{\frac{t+s}{4}}\left(\frac{x+y}{2}-\xi\right)\right\rangle \\
& =2 \tilde{u}\left(\frac{x+y}{2}, \frac{t+s}{4}\right) .
\end{aligned}
$$


Similarly we have

$$
\begin{aligned}
& {\left[\left(u \circ P_{1}\right) *\left(E_{t}(\xi) E_{s}(\eta)\right)\right](x, y)=\tilde{u}(x, t),} \\
& {\left[\left(u \circ P_{2}\right) *\left(E_{t}(\xi) E_{s}(\eta)\right)\right](x, y)=\tilde{u}(y, s),}
\end{aligned}
$$

where $\tilde{u}(x, t)$ are the Gauss transform of $u$. Thus the inequality (3.26) is converted to the stability problem

$$
\left|2 \tilde{u}\left(\frac{x+y}{2}, \frac{t+s}{4}\right)-\tilde{u}(x, t)-\tilde{u}(y, s)\right| \leq \epsilon\left(\mathcal{H}_{2 p}(x, t)+\mathcal{H}_{2 p}(y, s)\right) .
$$

Now applying Lemma 3.4 and letting $t \rightarrow 0^{+}$we have

$$
\|u-a \cdot x-c\| \leq 2 \epsilon \frac{4^{p}}{4^{p}-2}|x|^{2 p} .
$$

Since every locally integrable function $f(x)$ can be viewed as a distribution via the equation

$$
\langle f, \varphi\rangle=\int f(x) \varphi(x) d x
$$

we have the following stability theorem for locally integrable functions in almost everywhere sense.

Theorem 3.6. Let $\Omega_{1}, \Omega_{2} \subset \mathbb{R}^{n}$ such that $m\left(\mathbb{R}^{n} \backslash \Omega_{1}\right)=m\left(\mathbb{R}^{n} \backslash \Omega_{2}\right)=0$ and let $f: \mathbb{R}^{n} \rightarrow \mathbb{C}$ be locally integrable functions satisfying the inequality

$$
\left|2 f\left(\frac{x+y}{2}, \frac{t+s}{4}\right)-f(x, t)-f(y, s)\right| \leq \epsilon\left(\mathcal{H}_{2 p}(x, t)+\mathcal{H}_{2 p}(y, s)\right)
$$

for all $x \in \Omega_{1}, y \in \Omega_{2}$. Then there exist a unique $a \in \mathbb{C}^{n}$, complex constants $c$ and $\Omega \subset \mathbb{R}^{n}$ with $m\left(\mathbb{R}^{n} \backslash \Omega\right)=0$ such that

$$
\|f(x)-a \cdot x-c\| \leq 2 \epsilon \frac{4^{p}}{4^{p}-2}|x|^{2 p}
$$

for all $x \in \Omega$.

Acknowledgment. The first author was supported by the Research Foundation of Dongyang Mirae University and the second author was supported by the Korea Research Foundation Grant(KRF) grant funded by the Korea Government (MEST)(No. 2009-0063887).

\section{References}

[1] J.-H. Bae, D.-O. Lee, and W. G. Park, On the stability of the Jensen's equation in Banach modules, J. Appl. Math. Comput. 11 (2003), no. 1-2, 423-430.

[2] J. A. Baker, Distributional methods for functional equations, Aequationes Math. 62 (2001), no. 1-2, 136-142.

[3] P. W. Cholewa, Remarks on the stability of functional equations, Aequationes Math. 27 (1984), no. 1-2, 76-86.

[4] J. Chung, A distributional version of functional equations and their stabilities, Nonlinear Anal. 62 (2005), no. 6, 1037-1051.

[5] - Hyers-Ulam-Rassias stability of Cauchy equation in the space of Schwartz distributions, J. Math. Anal. Appl. 300 (2004), no. 2, 343-350. 
[6] Stability of functional equations in the space of distributions and hyperfunctions, J. Math. Anal. Appl. 286 (2003), no. 1, 177-186.

[7] J. Chung, S.-Y. Chung, and D. Kim, The stability of Cauchy equations in the space of Schwartz distributions, J. Math. Anal. Appl. 295 (2004), no. 1, 107-114.

[8] _ Une caractérisation de l'espace de Schwartz, C. R. Acad. Sci. Paris Sér. I Math. 316 (1993), no. 1, 23-25.

[9] Z. Gajda, On stability of additive mappings, Internat. J. Math. Math. Sci. 14 (1991), no. 3, 431-434.

[10] P. Găvruta, A generalization of the Hyers-Ulam-Rassias stability of approximately additive mappings, J. Math. Anal. Appl. 184 (1994), no. 3, 431-436.

[11] L. Hörmander, The Analysis of Linear Partial Differential Operator I, Springer-Verlag, Berlin-New York, 1983.

[12] D. H. Hyers, On the stability of the linear functional equations, Proc. Nat. Acad. Sci. USA 27 (1941), 222-224.

[13] S. M. Jung, Hyers-Ulam-Rassias stability of Jensen's equations and its application, Proc. Amer. Math. Soc. 126 (1998), no. 11, 3137-3143.

[14] Z. Kominek, On a local stability of the Jensen functional equation, Demonstratio Math. 22 (1989), no. 2, 499-507.

[15] T. Matsuzawa, A calculus approach to hyperfunctions III, Nagoya Math. J. 118 (1990), $133-153$.

[16] C.-G. Park, Generalized Jensen's equations in a Banach module, Southeast Asian Bull. Math. 29 (2005), no. 6, 1117-1123.

[17] Universal Jensen's equations in Banach modules over a $C^{*}$-algebra and its unitary group, Acta Math. Sin. (Engl. Ser.) 20 (2004), no. 6, 1047-1056.

[18] _ Generalized Jensen's equations in Banach modules over a unital $C^{*}$-algebra, Southwest J. Pure Appl. Math. 2002 (2002), no. 2, 52-63.

[19] C.-G. Park and W.-G. Park, On the stability of the Jensen's equation in a Hilbert module, Bull. Korean Math. Soc. 40 (2003), no. 1, 53-61.

[20] _ On the Jensen's equation in Banach modules, Taiwanese J. Math. 6 (2002), no. 4, 523-531.

[21] Th. M. Rassias, On the stability of functional equations in Banach spaces, J. Math. Anal. Appl. 251 (2000), no. 1, 264-284.

[22] _ On the stability of the linear mapping in Banach spaces, Proc. Amer. Math. Soc. 72 (1978), no. 2, 297-300.

[23] L. Schwartz, Théorie des Distributions, Hermann, Paris, 1966.

[24] S. M. Ulam, Problems in Modern Mathematics, Chapter VI, Wiley, New York, 1964.

\section{Eun Gu LeE}

Department of Internet Business

Dongyang Mirae University

SEOUL 152-714, KorEA

E-mail address: eglee@dongyang.ac.kr

JAEYOUNG Chung

Department of Mathematics

KUNSAN NATIONAL UNIVERSITY

Kunsan 573-701, Korea

E-mail address: jychung@kunsan.ac.kr 